\title{
Statistical properties of concentration fluctuations in two merging plumes
}

\author{
D. Contini · A. G. Robins · P. Hayden
}

Received: 23 May 2013 / Accepted: 9 December 2013

C Springer Science+Business Media Dordrecht 2013

\begin{abstract}
The statistics of the fluctuating concentration field within a plume is important in the analysis of atmospheric dispersion of toxic, inflammable and odorous gases. Previous work has tended to focus on concentration fluctuations in single plumes released in the surface layer or at ground level and there is a general lack of information about the mixing of two adjacent plumes and how the statistical properties of the concentration fluctuations are modified in these circumstances. In this work, data from wind tunnel experiments are used to analyse the variance, skewness, kurtosis, intermittency, probability density function and power spectrum of the concentration field during the mixing of two identical plumes and results are compared with those obtained for an equivalent single plume. The normalised variance, skewness and kurtosis on the centre-lines of the combined plume increase with distance downwind of the stack and, in the two-source configuration, takes lower values than those found in the single plumes. The results reflect the merging process at short range, which is least protracted for cases in which the sources are in-line or up to $30^{\circ}$ off-line. At angles of $45^{\circ}$ and more, the plumes are effectively side-by-side during the merging process and the interaction between the vortex pairs in each plume is strong. Vertical asymmetry is observed between the upper and the lower parts of the plumes, with the upper part having greater intermittency (i.e. the probability that no plume material is present) and a more pronounced tail to the concentration probability distribution. This asymmetry tends to diminish at greater distances from the source but occurs in both buoyant and neutral plumes and is believed to be associated with the 'bending-over' of the emission in the cross-flow and the vortex pair that this generates. The results allowed us to identify three phases in plume development. The first, very near the stack, is dominated by turbulence generated within the plume and characterised by concentration spectra with distinct peaks corresponding to scales comparable with those of the counter-rotating vortex pair. A second phase follows at somewhat greater distances
\end{abstract}

D. Contini $(\bowtie)$

CNR-ISAC, Istituto di Scienze dell' Atmosfera e del Clima, Str. Prv. Lecce-Monteroni Km 1.2,

73100 Lecce, Italy

e-mail: d.contini@isac.cnr.it

A. G. Robins · P. Hayden

EnFlo, FEPS, University of Surrey, Guildford GU2 7XH, UK 
downwind, in which there are significant contributions to the concentration fluctuations from both the turbulence internal to the plume and the external turbulence. The third phase is one in which the concentration fluctuations appear to be controlled by the external turbulence present in the ambient flow.

Keywords Concentration fluctuations - Two-plume interaction · Intermittency · Wind tunnel simulations

\section{Introduction}

The response of biological systems to toxic gases, vapours and odours is generally nonlinear, making it susceptible to concentration fluctuations. Short-term concentration fluctuations, caused by turbulent mixing processes, can be important in determining the hazard caused by industrial releases and accidental emissions, as well as for understanding the behaviour of reacting plumes. In urban areas, emissions from vehicle tailpipes undergo very rapid mixing in the turbulent wake of the vehicles and pass through a number of stages in a few seconds [1]. The dynamics of concentration fluctuations is also important in the study of nanoparticle formation and growth (secondary aerosol) because this is a competitive effect in which the number concentration of particles is increased by the nucleation process but is reduced by coagulation, which is strongly dependent on instantaneous concentration levels [2]. This means that concentration fluctuations in developing plumes is a topic that needs to be understood in detail and then modelled. Analysis of the fluctuating concentration field has been based on full scale experiments [3-5], small scale models in wind tunnels [6-10] and in water channels $[11,12]$. Models have been developed to evaluate the intensity of fluctuations and the statistics of exceeding specific concentration thresholds [13-18]. Remote-sensing with LIDAR systems has also been used to characterize fluctuations in full scale plumes $[19,20]$. In Fackrell and Robins [6] wind tunnel simulations were used to investigate the effect of source size on concentration fluctuations in single plumes. In Finn et al. [21] field tracer experiments were used to study concentration fluctuations in plumes dispersing in the urban atmosphere and Pavageau and Schatzmann [22] carried out related work for street canyons based on wind tunnel experiments.

In general terms, laboratory small scale models suffer from the disadvantage that large scale fluctuations in wind directions influence the meandering of plumes, can not be adequately simulated, so that measurements are representative of relatively short time averages at full scale (from a few minutes to about half an hour). Previous research work has mainly concentrated on single plumes, whether from elevated or ground level sources, even though industrial emissions are often characterised by multiple releases from a number of stacks. This is a typical configuration for waste incinerators, power plants and petrochemical complexes. The mixing of plumes in such circumstances involves strong plume to plume interactions, especially for favourable wind directions, that distort cross-sectional shapes and generate extra-rise or "downwash" in comparison with single source reference cases [23]. Releases from multiple stacks have been analysed in some detail to understand the influence of their interactions on the trajectory [24-28], the structure and shape of the combined plumes [29,30], and on the flow features [31]. Nevertheless, a comprehensive analysis of the effect of the mixing process on the statistical properties of the concentration fluctuations is largely absent.

The objective of the present work was therefore to study the changes in the statistical properties (i.e. concentration probability density functions, power spectra and the first four concentration moments) of the fluctuating concentration field induced by the mixing 
processes between two identical plumes at different orientations to the approaching flow. The equivalent single plume provides reference conditions against which changes can be measured. Some experiments with neutral releases (i.e. without buoyancy) provide further reference cases for assessing the role of buoyancy. Finally, the observations are contrasted with the well established characteristics of truly passive dispersion (i.e. emissions with no significant buoyancy or momentum effects). In this way an understanding is built of the role of emission conditions and plume merger in buoyant plumes.

\section{Experimental set-up and equipment}

The experiments were carried out in the EnFlo (University of Surrey) environmental wind tunnel facility ( $20 \mathrm{~m}$ long working section, $3.5 \mathrm{~m}$ wide and $1.5 \mathrm{~m}$ tall), operated in neutral conditions with a free-stream velocity, $U_{\text {ref }}=2 \mathrm{~m} / \mathrm{s}$, maintained constant through a softwarecontrolled feedback system. A schematic diagram of the wind tunnel set-up is shown in Fig. 1a. The $\mathrm{H}=1 \mathrm{~m}$ deep boundary-layer was artificially generated using five $1.2 \mathrm{~m}$ tall Irwin spires [32] located at $1 \mathrm{~m}$ from the tunnel inlet, a $190 \mathrm{~mm}$ tall barrier placed $0.77 \mathrm{~m}$ from the inlet and a staggered distribution of plate-shaped roughness elements $(20 \mathrm{~mm}$ tall and $80 \mathrm{~mm}$ wide) covering the floor of the tunnel with a lateral spacing of $240 \mathrm{~mm}$.

Two stacks, both of height, $\mathrm{h}=300 \mathrm{~mm}(\mathrm{~h} / \mathrm{H}=0.3)$, with an internal diameter, $\mathrm{D}=5 \mathrm{~mm}$ $(\mathrm{D} / \mathrm{h}=0.017)$ and a separation, $\mathrm{d}=70 \mathrm{~mm}(\mathrm{~d} / \mathrm{D}=14)$, were used for two-source configurations. This separation was chosen because it provides a relatively small effect on plume rise and a relatively long mixing phase of the two plumes [23], thereby providing scope to study in detail the effects of the mixing on the concentration field. Different angles, $\phi$, of alignment between the stacks and the cross-flow were used. Figure 1b illustrates the set-up and includes the definition of the alignment angle, $\phi$, and the origin of the co-ordinate system. For comparison purposes, single source cases were also studied with $\mathrm{D}=5$ and $7.1 \mathrm{~mm}$ (note that $7.1=5 \sqrt{ } 2$, equivalent to two perfectly merged plumes). In what follows, the term 'centre-line' is taken to be the line $y=0, z=z_{P}$ for the single plume and $y=y_{\text {mid }}, z=z_{P}$ for the plume pair; $z_{P}$ is the plume height, which is a function of $x$, and $y_{\text {mid }}$ is the mid-way position between the two sources.

Boundary-layer properties were measured with a two-component LDA system, based on a DANTEC BSA 57N11 burst spectrum analyzer. The flow was seeded using a solution of sugar and water in a commercial haze generator placed just outside the tunnel inlet. The seeding was distributed in an acceptably uniform way in the measurement area and the LDA was operated at an average acquisition frequency of about $100 \mathrm{~Hz}$. Velocity and turbulence measurements were taken on the tunnel centre-line at three distances, $x$, downwind of the source position (i.e. $\mathrm{x}=0, \mathrm{x}=0.5$ and $\mathrm{x}=1.1 \mathrm{~m}$ ). The mean velocity profile is plotted in Fig. 1c, this being the average of the profiles at the three positions. It was characterised by a logarithmic form up to about $\mathrm{z}=0.5 \mathrm{~m}$, with $\mathrm{u}^{*} / \mathrm{U}_{\text {ref }}=0.055$ and $\mathrm{z}_{\mathrm{o}}=1.6 \mathrm{~mm}$ (see Fig. 1c). This was also the height range in which the Reynolds stress $\left\langle u^{\prime} w^{\prime}\right\rangle$ was approximately constant.

The profiles of the dimensionless turbulence intensities $\left(\sigma_{\mathrm{u}}, \sigma_{\mathrm{w}}\right)$ and the Reynolds shear stress, $\left\langle\mathrm{u}^{\prime} \mathrm{w}^{\prime}\right\rangle$, are plotted, respectively, in Fig. 2a, b in terms of their spatial averages over the three positions analysed; error bars (the standard deviation of the results) are included. The average power spectra of the longitudinal and vertical turbulence components are shown in Fig. 2c. Power spectra were obtained at the heights of the developing plumes (i.e. $z$ between 0.3 and $0.4 \mathrm{~m}$ ) and the figure shows the spatial averaged spectra at the three positions $\mathrm{x}$ and their variability (the standard deviation, represented as dashed lines). In the figures, $\mathrm{f}$ denotes 
Fig. 1 a Schematic view of experimental configuration in the EnFlo wind tunnel. b Schematic of the two-stack configuration with co-ordinate and orientation definitions. c Mean velocity vertical profile, obtained as a spatial average at $x=0,500$ and $1,100 \mathrm{~mm}$. The graph includes a logarithmic fit and error bars evidencing spatial variability (as $1 \mathrm{SD}$ ) in the plume development zone

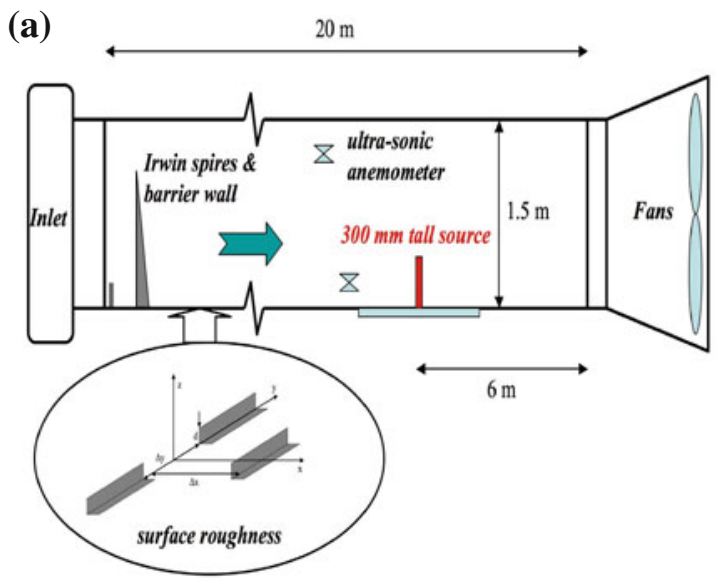

(b)
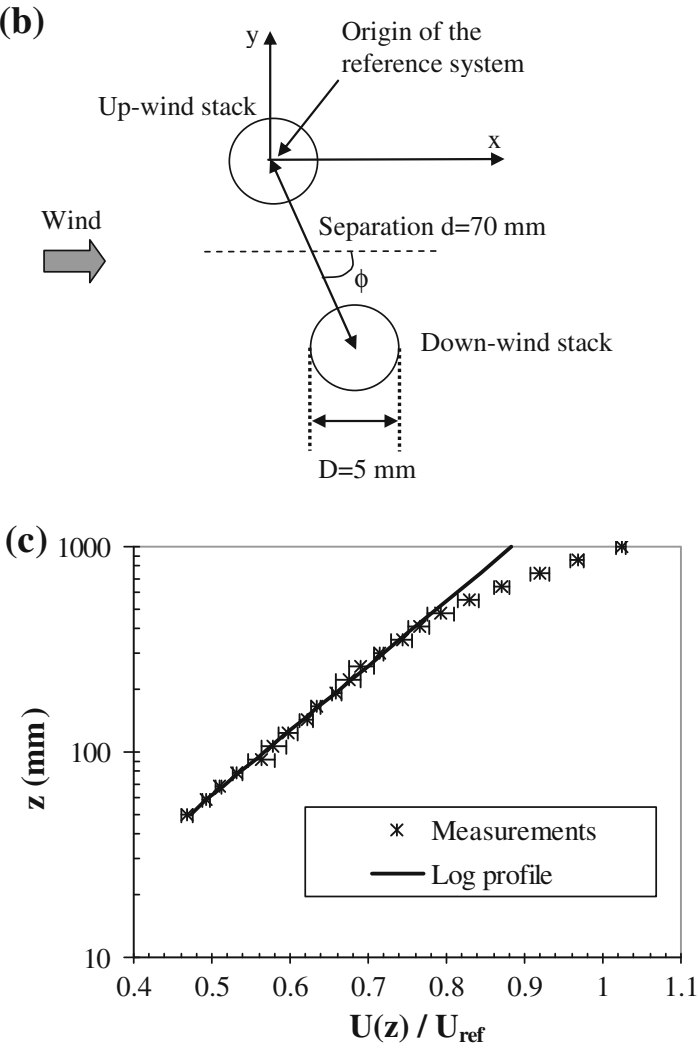

the frequency (Hz) and $n=f z / U(z)$ the normalised (i.e. dimensionless) frequency. Some flow measurements were also performed off-axis (with respect to the tunnel centre-line), exploring the area in which simulated plumes develop. Results (not shown) indicate that the flow was sufficiently homogeneous in the measurement area for the purposes of this work with lateral variation of $U$ at the source height limited within $4 \%$. Based on the turbulence intensity levels and the 0.23 exponent of a power law fit to the mean velocity profile, the 
Fig. 2 a Vertical profiles of longitudinal and vertical turbulence. b Vertical profile of Reynolds stress. c Power spectra of horizontal and vertical wind velocity fluctuations.

Measurements are a spatial average of profiles measured at $\mathrm{x}=0,500$ and $1,100 \mathrm{~mm}$ and error bars represent the spatial variability in term of $1 \mathrm{SD}$
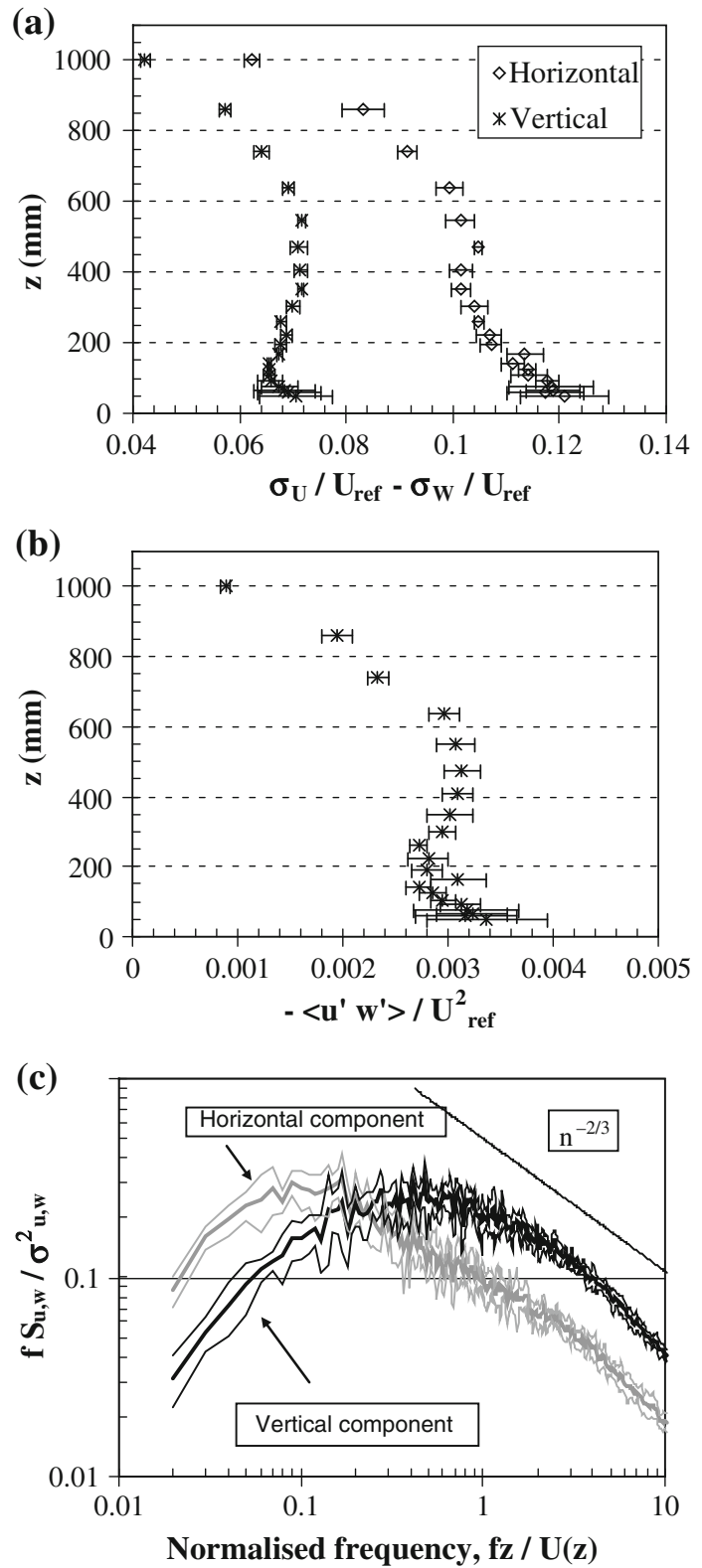

boundary-layer could be considered as a rough wall, neutral boundary layer. The increase in the normal and shear stresses near the ground, along with the greater variability between the results at the three locations, is probably due to 3-D effects in the vicinity of individual roughness elements.

Buoyant emissions with a stack flow-rate of $Q=101 /$ min were created by a three gas mixture of helium, air and propane in appropriate proportions. The propane trace gas concentration (typically between 0.25 and $2.4 \%$ at the source) was changed from case to case as 
required to optimise the response of the detector used; air and helium were mixed in order to provide a buoyant plume with a density, $\rho_{\mathrm{s}}$, chosen in such a way that the density difference, $\Delta=\rho_{a}-\rho_{\mathrm{s}}$ (with $\rho_{\mathrm{a}}$ the environmental air density) was maintained at $\Delta \rho / \rho_{\mathrm{a}}=0.79$. All gas flow-rates were controlled and measured by three HI-TEC thermal, mass-flow regulators that had been previously calibrated with both a volumetric counter gas-meter and a bubble flow-meter. Some cases of neutrally buoyant plumes (i.e. $\rho_{\mathrm{a}}=\rho_{\mathrm{s}}$ but with upwards momentum, so not a passive emission) were also studied for comparative purposes and used to determine the differences between buoyant and neutral plume behaviour. These were all single stack cases with $\mathrm{D}=7.1 \mathrm{~mm}$ and a flow-rate $\mathrm{Q}=20$ or $30 \mathrm{l} / \mathrm{min}$. Table 1 summarises the source properties of the plumes simulated in the experiments, information that could be useful when making comparison with other experiments. Specifically, Table 1 includes the source Reynolds number $\operatorname{Re}=\frac{W D}{v}$, the densimetric Froude number $F_{r}=W / \sqrt{g \frac{\Delta \rho}{\rho_{\mathrm{a}}} \mathrm{D}}$, the momentum flux ratio $\rho_{\mathrm{s}} \mathrm{W}^{2} / \rho_{\mathrm{a}} \mathrm{U}^{2}$, the momentum flux $\mathrm{F}_{\mathrm{M}}=\frac{\rho_{\mathrm{s}}}{\rho_{\mathrm{a}}} \mathrm{W}^{2} \mathrm{r}^{2}$, the buoyancy flux $F_{B}=\operatorname{gr}^{2} \mathrm{~W} \frac{\left(\rho_{\mathrm{a}}-\rho_{\mathrm{s}}\right)}{\rho_{\mathrm{a}}}$, the buoyancy length scale $\mathrm{L}_{\mathrm{B}}=\frac{\mathrm{F}_{\mathrm{B}}}{\mathrm{U}^{3}}$ and the momentum length scale $\mathrm{L}_{\mathrm{M}}=\frac{\sqrt{\mathrm{F}_{\mathrm{M}}}}{\mathrm{U}} ; \mathrm{W}$ is the exit velocity at the stack calculated assuming a uniform profile, $\mathrm{W}=\frac{4 \mathrm{Q}}{\pi \mathrm{D}^{2}}$, and $\mathrm{g}$ the gravitational acceleration $\left(\mathrm{g}=9.81 \mathrm{~ms}^{-2}\right)$.

Point concentration measurements were carried out by using two FFIDs (fast FIDs, HRF 400 from Cambustion Ltd.) placed on the traversing system of the wind tunnel, sampling at the same position $\mathrm{z}$ above the wind tunnel floor and distance $\times$ downwind of the source, and fixed $50 \mathrm{~mm}$ apart in the y direction (i.e. cross-wind). The FFIDs were equipped with a $250 \mathrm{~mm}$ long and $1.2 \mathrm{~mm}$ internal diameter tube for calibration purposes around a second smaller tube that was the actual sampling tube (internal diameter $0.305 \mathrm{~mm}$, estimated sample flow-rate $1.24 \mathrm{mg} / \mathrm{s}$ ). The FFIDs response time measured during laboratory tests by their response to a large step concentration change was about $5 \mathrm{~ms}$ (between 5 and $95 \%$ of a step change in concentration). Concentration maps at different distances downwind of the sources were obtained on a regular grid using a number of points between 130 and 340 per cross section. At each position the outputs from the FFIDs were recorded at a sampling frequency of $500 \mathrm{~Hz}$ for about $3.5 \mathrm{~min}$. The stability and repeatability of the FFID calibrations were good throughout the measurements (calibration checks were performed every $1-2 \mathrm{~h}$ of continuous operation), with output voltage changes between one calibration and the successive usually $<0.5 \%$ and changes through a day usually $<1 \%$.

\section{Discussion of results}

\subsection{Stationarity of concentration time-series}

The stationarity of the first four moments of concentration (i.e. mean, variance, skewness and kurtosis) was investigated using a methodology generally applied in full scale micrometeorology $[33,34]$. Each concentration time-series $(3.5 \mathrm{~min}$ long) was divided in four segments (each $52.5 \mathrm{~s}$ long) and, for each segment, i, the first four concentration moments were evaluated. These were expressed as:

the average concentration $\langle\mathrm{c}\rangle_{\mathrm{i}}$,

the normalised variance: $V_{i}=\frac{\left\langle\left(c-\langle c\rangle_{i}\right)^{2}\right\rangle_{i}}{\langle c\rangle_{i}^{2}}=\frac{\left(\sigma_{c}\right)_{i}^{2}}{\langle c\rangle_{i}^{2}}$, 


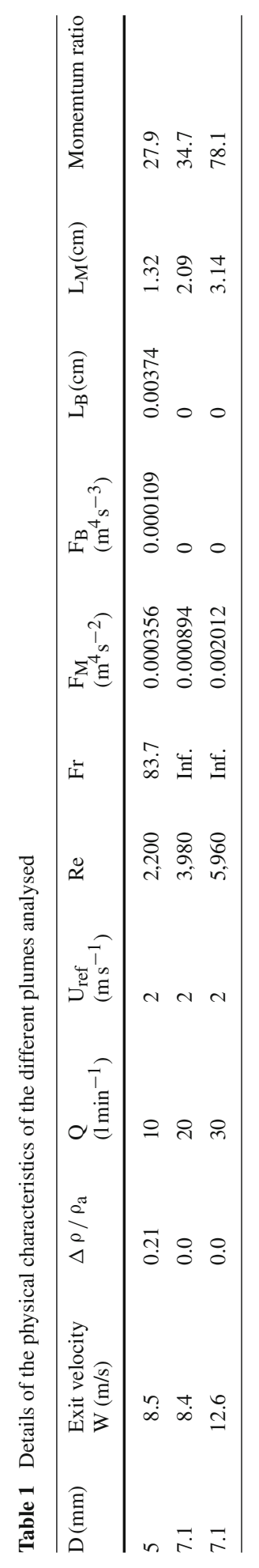


Fig. 3 Evaluation of stationarity of the statistical parameters. a Relative variability for the first four concentration moments on the centreline $(\mathrm{y}=0$ and $\mathrm{z} / \mathrm{H}=0.375$ ) for a single plume at $\mathrm{x} / \mathrm{H}=0.3$. b Relative variability for the first four concentration moments for a two-stack configuration with $\phi=0^{\circ}$ measured at $\mathrm{x} / \mathrm{H}=0.15$, $\mathrm{y} / \mathrm{H}=0.04, \mathrm{z} / \mathrm{H}=0.424$
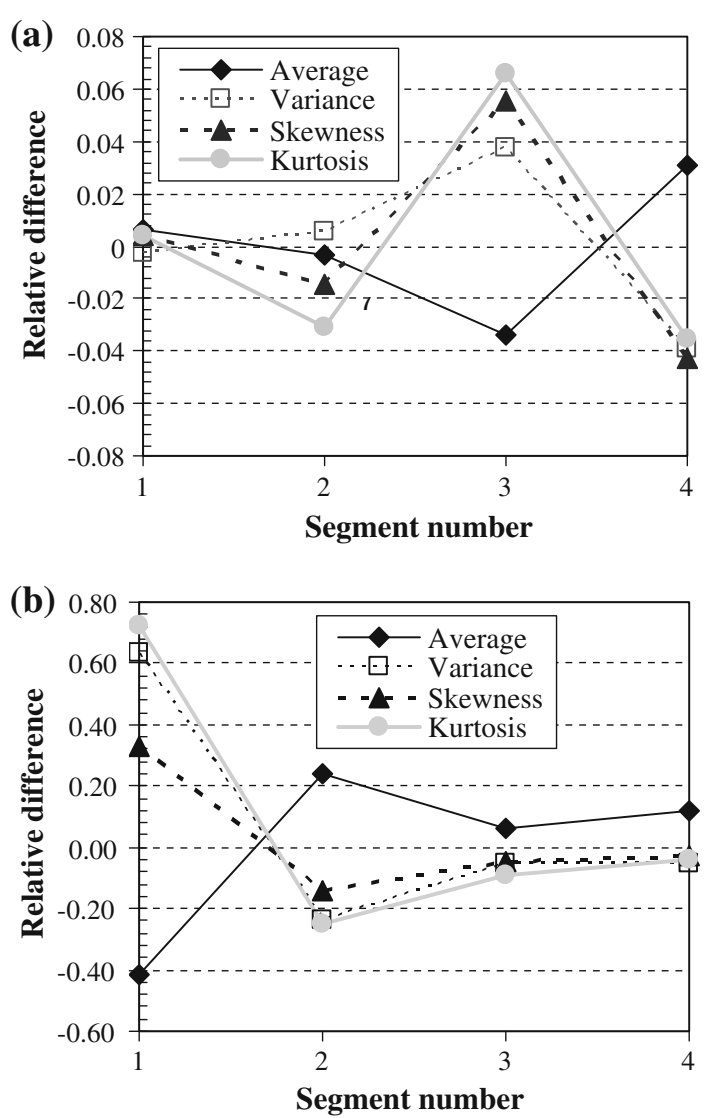

$$
\begin{aligned}
& \text { the skewness: } S_{i}=\frac{\left\langle(\mathrm{c}-\langle\mathrm{c}\rangle)^{3}\right\rangle_{\mathrm{i}}}{\left(\sigma_{\mathrm{c}}\right)_{\mathrm{i}}^{3}} \\
& \text { and the kurtosis: } \mathrm{K}_{\mathrm{i}}=\frac{\left\langle(\mathrm{c}-\langle\mathrm{c}\rangle)^{4}\right\rangle_{\mathrm{i}}}{\left(\sigma_{\mathrm{c}}\right)_{\mathrm{i}}^{4}} \text {. }
\end{aligned}
$$

The symbol \langle\rangle$_{\mathrm{i}}$ indicates a time average over the segment $\mathrm{i}$ and the symbol \langle\rangle a time average of the whole time-series. A stationarity index, $\mathrm{S}_{\mathrm{i}}^{\mathrm{p}}$, was evaluated for each moment, $\mathrm{p}$, and each interval, $\mathrm{i}$, in a specific time-series from the difference between the $\mathrm{p}$-moment calculated over the period $i$ and that calculated for the whole time-series. $S_{i}^{p}$ was obtained as the ratio of this difference and the p-moment calculated over the all time-series. A specific time-series was considered stationary if $S_{i}^{p}$ was $<0.5$ for all values of $i$ and $p$. In full scale micrometeorological measurements a variability of 0.5 is generally used as the threshold for the identification of non-stationary cases in fundamental research applications [34]. In Fig. 3a an example set of $S_{i}^{p}$ values is shown for a stationary case (single plume at $x=300 \mathrm{~mm}$ ) and in Fig. 3b an example of a non-stationary time series is shown, in which $S_{1}^{2}$ and $S_{1}^{4}$ are lager than 0.5 (two-source configuration with $\phi=0^{\circ}$ at $\mathrm{x}=150 \mathrm{~mm}$ ).

A total of 5,324 time-series was analysed in this manner and $92.4 \%$ were found to be stationary. In $71.4 \%$ of the cases $\mathrm{S}_{\mathrm{i}}^{\mathrm{p}}$ was $<0.25$. The $7.6 \%$ of non-stationary cases were predominantly found at plume boundaries where meandering of the plume and large scale 
vertical fluctuations create significant concentration fluctuations at relatively large temporal scales that can result in non-stationary signals unless the averaging time is suitably extended. This is compatible with the observations reported in full scale experiments [5] and in other wind tunnel experiments [10] that illustrate how the high intermittency observed at the edge of dispersing urban plumes can lead to difficulties in achieving representative statistics for concentration fluctuations, especially in full scale experiments.

\subsection{Intermittency of the concentration time series}

The intermittency, I, of the concentration signal was evaluated as $\mathrm{I}=1-\gamma$, where $\gamma$ is defined as the fraction of measurement time in which significantly "non-zero" concentrations are observed. In practice, this means the fraction of time in which the measured concentration is above a certain (small) threshold; in the present work the threshold for calculating $\gamma$ was chosen as the maximum of $1 \mathrm{ppb}$ and $\alpha\langle\mathrm{c}\rangle$, with $\alpha=0.05$ and $\langle\mathrm{c}\rangle$ the average concentration. This was selected because our analysis showed that a $1 \mathrm{ppb}$ concentration was generally not distinguishable from background concentrations in the wind tunnel. Fast instrument response is important in the evaluation of I. The interface separating contaminant and clean air regions contains variations at scales smaller than those measurable by the FFID detector because of the instrument's limited time response. These fluctuations therefore appear mixed and convoluted in the measurements, implying that the intermittency results are an upper bound to the true values that would be obtained by a detector able to resolve all scales [4]. Because the concentration is zero outside the plume, conditionally averaged statistical parameters (i.e. in-plume data) can be obtained from conventional measurements and knowledge of I. This has not been done in the present work because the goal was best served by working with complete statistics. The most interesting form of conditioned measurements would probably be in-plume at fixed locations with respect to the instantaneous plume centre, but such data was not available.

The absolute value of I depends on source size, and the effect of size is known to be greater for elevated than ground-based plumes. However, the general features of the variation of I with $\mathrm{x}$ along the centre-line of an elevated plume are similar, no matter what the source size might be. $\mathrm{I}$ is zero $(\gamma=1)$ at the source and then rapidly increases to a maximum value $(\gamma<1)$, which is source-size dependent, before slowly decreasing again $(\gamma$ increasing; [7]). Whether or not I returns to zero $(\gamma=1)$ far downwind remains a moot point. In the experiments, the momentum and buoyancy length scales of the plume motion were such that the majority of the plume rise occurred in the region where I was increasing.

In Fig. 4a the intermittency, measured on the centreline of (1) the plume from a single source and (2) the combined plume from two sources at a range of angles, $\phi$, is plotted as a function of $\mathrm{x}$. The intermittency in the single and two-source plumes with $\phi<30^{\circ}$ increased monotonically with $\mathrm{x}$. The variations is more complex for two-source plumes with $\phi \geq 30^{\circ}$, where I initially decreases to a minimum at around $\mathrm{x} / \mathrm{H}=0.2-0.3$ and then increases again. The dependence on the angle $\phi$ arises because at small values of $\phi$ the lower plume from the more downwind source, which is smaller and more concentrated, is "guided" into the centre of the larger upper plume by the counter-rotating vortex pair. In contrast, mixing is a slower and less 'efficient' process for large values of $\phi$ as the two vortex systems interact with opposing signs of vorticity. At large values of $\mathrm{x}$, intermittency for the two-source configurations is less than for the corresponding single source cases for all values of $\phi$. The present results cover the range $\mathrm{x} / \mathrm{H}=0.1-0.6$, which is the region in which $\mathrm{I}$ is increasing $(\gamma$ decreasing $)$ in the results of Fackrell and Robins [7] and where I decreases ( $\gamma$ increases) with increasing source size. The observations in Fig. 4a follow this trend as the interacting plumes are locally larger 

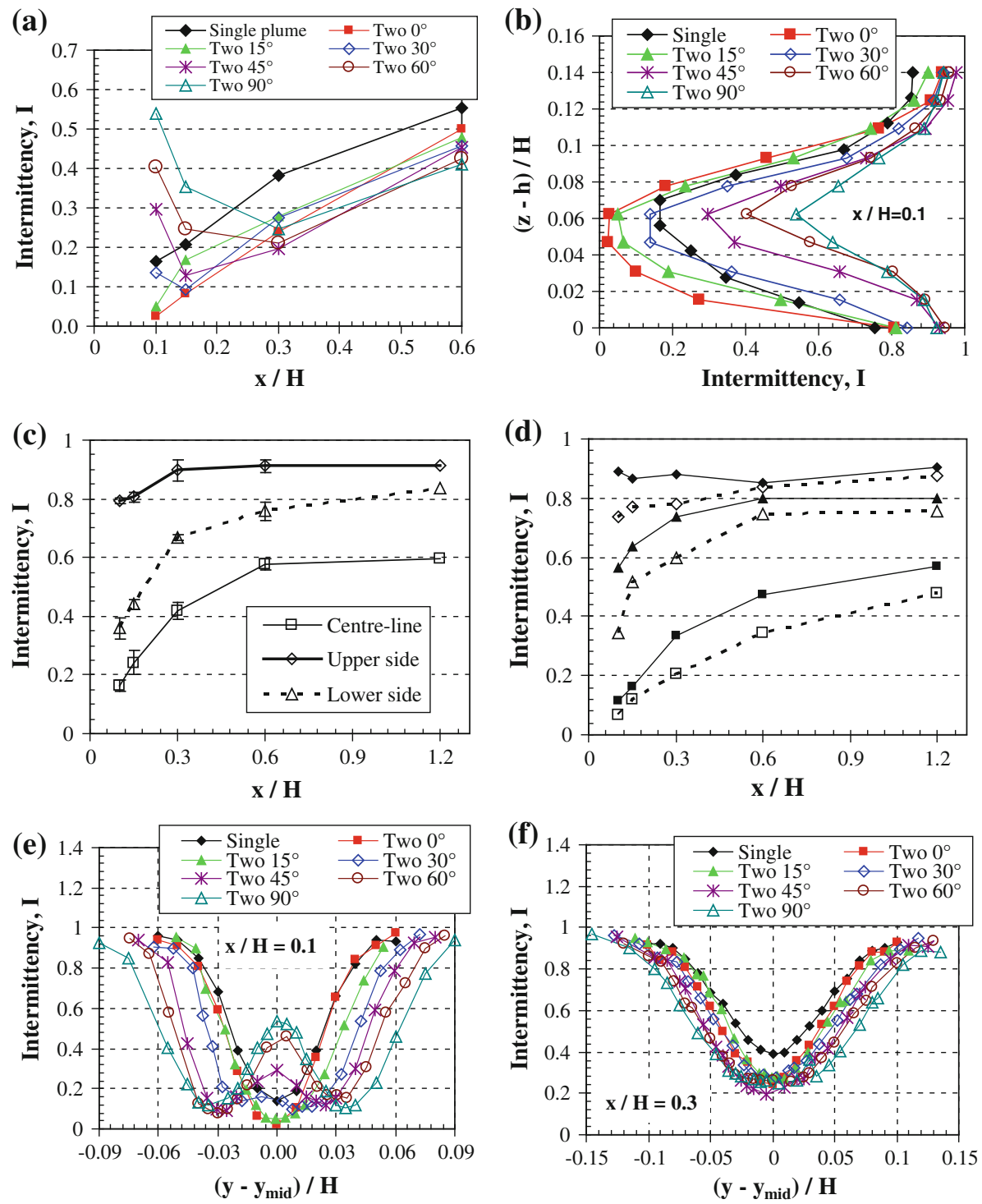

Fig. 4 a Intermittency along the plume centre-lines for different configurations of sources. b Vertical profiles of intermittency at $\mathrm{y}=\mathrm{y}_{\text {mid }}$ for different configuration of sources. $\mathbf{c}$ Intermittency on the plume centreline and at the lower and upper sides of the plume for the single stack buoyant release with $\mathrm{D}=5 \mathrm{~mm}$ and $\mathrm{Q}=101 / \mathrm{min}$. Error bars represents variability in nominally identical conditions. $\mathbf{d}$ Intermittency on the plume centre-line (squares) and at the lower (triangles) and upper sides (diamonds) of the plume for a single stack neutral release with $\mathrm{D}=7.1 \mathrm{~mm}$ and $\mathrm{Q}=20 \mathrm{l} / \mathrm{min}$ (filled marks) and $\mathrm{Q}=301 / \mathrm{min}$ (empty marks). e Horizontal profiles of intermittency at $\mathrm{z}=\mathrm{z}_{\mathrm{p}}$ and $\mathrm{x} / \mathrm{H}=0.1$ for different configuration of sources. $\mathbf{f}$ Horizontal profiles of intermittency at $\mathrm{z}=\mathrm{z}_{\mathrm{p}}$ and $\mathrm{x} / \mathrm{H}=0.3$ for different configuration of sources

than the single plume and, consequently, I is smaller. Behaviour at very short range, less than about $\mathrm{x} / \mathrm{H}=0.3$, reflects the fact that the two plumes have not significantly mixed over this fetch when $\phi \geq 45^{\circ}$, something that can be clearly seen in the lateral intermittency profiles (Fig. 4e, f). 
Vertical profiles of intermittency, measured in the centre-plane $\left(y=y_{\text {mid }}\right)$ of the merging plumes at $\mathrm{x} / \mathrm{H}=0.1$, are plotted in Fig. $4 \mathrm{~b}$ for all configurations. The results show that vertical asymmetry is present for both the single and two-source configurations. The dependence on the angle $\phi$ arises because the nature of the merging process changes with $\phi$, as discussed above. Figure $4 \mathrm{c}$ shows intermittency in the centre-plane of the single plume as a function of $\mathrm{x}$ at three different positions: the centre-line, a characteristic location on the upper edge of the plume and one on the lower edge. These locations are the points in the centre-plane of the plume (i.e. on $y=0$ in the case of a single plume) where the concentration is approximately equal to $15 \%$ of the centre-line concentration. Error bars, shown as 1 SD, were derived from several repeated experiments in nominally identical conditions. The results indicate that there is significant vertical asymmetry, with the lower part of the plume having, on average, lower intermittency than the upper part. The vertical asymmetry might well be an effect related to buoyancy because, in a buoyant plume, the lower part is stably stratified in contrast to the upper part that is unstably stratified. However, this behaviour is also observed in neutrally buoyant plumes (i.e. with $\rho_{\mathrm{a}}=\rho_{\mathrm{s}}$ ) as shown in Fig. $4 \mathrm{~d}$ (the equivalent to Fig. $4 \mathrm{c}$ for the plume from a single source with $\mathrm{D}=7.1 \mathrm{~mm}$, neutral buoyancy and two emission rates, $\mathrm{Q}=20$ and 301/min). Of course, this is not a 'passive' plume and the bending-over of the upwards emission rather than the buoyance may still be sufficient to generate asymmetry due to the internal dynamics of the plume. Furthermore, and in line with this argument, I is greater for the lower emission rate. It is possible that dispersion in the wind shear $[35,36]$ contributes to the vertical asymmetry in the plume. However, the asymmetry is also visible very near the stack (at $\mathrm{x} / \mathrm{H}=0.1$ ) where the size of the plume is limited and the wind velocity changes by more than about $6 \%$ through the plume and the turbulence intensity by $<2 \%$. Furthermore, asymmetry due to plume rise is also clear in previous experiments on plume rise in uniform, laminar flow conditions [28].

Many of the features noted above are made more clear by the lateral distributions of intermittency plotted in Fig. 4e, f. Profiles of intermittency at $\mathrm{z}=\mathrm{z}_{\mathrm{p}}$ and $\mathrm{x} / \mathrm{H}=0.1$ are shown in Fig. 4e for the different source configurations, whereas Fig. 4f shows equivalent data for $\mathrm{x} / \mathrm{H}=0.3$. The intermittency distribution observed in a single developing plume is symmetrical, with I at minimum on the centre-line and zero at the edges and this is also the case for the combined plumes from two sources at $\mathrm{x} / \mathrm{H}=0.1$ when $\phi \leq 15^{\circ}$. and for all $\phi$ at $\mathrm{x} / \mathrm{H}=0.3$. However, at $\mathrm{x} / \mathrm{H}=0.1$ there are two off centre-line minima and a local maximum at $y=0$ for $\phi \geq 45^{\circ}$, reflecting the fact that the two plumes are far from fully merged at this location. Further downwind, at $\mathrm{x} / \mathrm{H}=0.3$, merger seems to be far more advanced at the most obvious effect of the orientation is seen in the width of the distributions, being narrowest for $\phi=0^{\circ}$ and broadest for $\phi=90^{\circ}$. A further manifestation of the mixing processes in seen in Fig.4b, where the profile for $\phi=0^{\circ}$ is simply deeper than the others but shows no other sign of the two merging plumes. Figure 5 shows the ratio $\sigma_{\mathrm{c}} /\langle\mathrm{c}\rangle$ in the centre-plane of the single plume as a function of $\mathrm{x}$ at three different locations in the plume: on the plume centre-line and at characteristic locations in the upper and lower edges. The results show that $\sigma_{\mathrm{c}} /\langle\mathrm{c}\rangle$ is increased downwind of the stack and was lower on the centre-line than at the edges. The difference between the values in the upper and lower edges of the plume is modest but consistent (with the lower side showing lower $\sigma_{\mathrm{c}} /\langle\mathrm{c}\rangle$ ) though the error bars reported in the figure generally span both sets of data.

\subsection{Concentration probability density functions and power spectra}

The probability density function (PDF) of instantaneous concentrations fluctuations has been studied extensively (e.g. by Fackrell and Robins [7]); it is generally expressed as $\operatorname{PDF}(\chi)$, 
Fig. $5 \sigma_{c} /\langle c\rangle$ as a function of $x$ on the plume centre-line and in the lower and upper edges of the plume; single stack buoyant release with $\mathrm{D}=5 \mathrm{~mm}$ and $\mathrm{Q}=101 / \mathrm{min}$

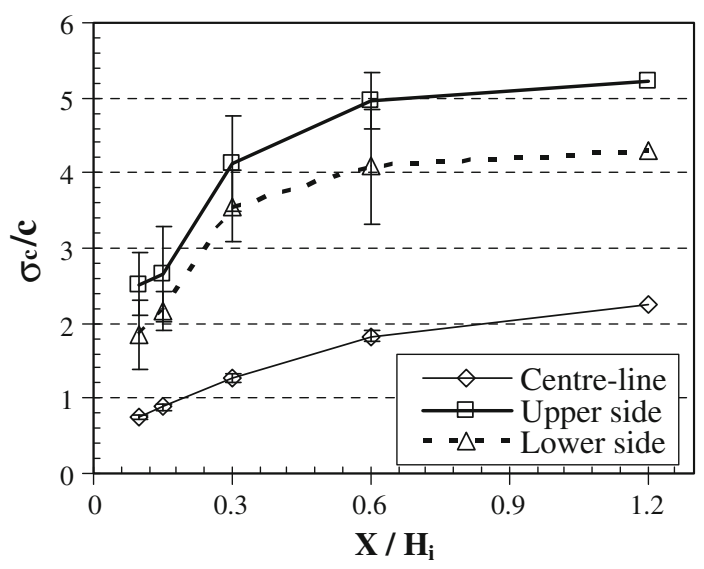

using the dimensionless variable:

$$
\chi=\frac{\mathrm{c}-\langle\mathrm{c}\rangle}{\sigma_{\mathrm{c}}}
$$

normalized so that:

$$
\int_{-\infty}^{\infty} \operatorname{PDF}(x) \mathrm{d} x=1 .
$$

The probability density function is plotted in Fig. 6 at the three locations in the plume cross-section defined previously and used in Fig. $4 \mathrm{c}$. The sharp peaks at $\mathrm{c}=0$ simply reflect the intermittency of the concentration field and are not an artefact of instrument noise. The associated power spectra, $\mathrm{Sn}$, of the concentration fluctuations are shown in Fig. 6; these are plotted in normalised form as: $\mathrm{Sn}=\mathrm{f} \frac{\mathrm{S}_{\mathrm{C}}}{\sigma_{\mathrm{c}}^{2}}$, where $\mathrm{S}_{\mathrm{C}}$ is the spectrum in standard units and $\mathrm{f}$ the natural frequency. The normalized frequency is $\mathrm{n}=\frac{\mathrm{fz}}{\mathrm{U}(\mathrm{z})}$, with $\mathrm{U}(\mathrm{z})$ the wind speed at the height $\mathrm{z}$. Included in the figure is the $\mathrm{n}^{-2 / 3}$ line to indicate the inertial sub-range. All spectra show a limited and in some cases the absence of the inertial sub-range observed in other wind tunnel data [7,37], or in full scale experiments [38]. This could be due to the relatively low Reynolds numbers [39] of the present experiments. Low wind speeds and low emission velocity from the stack must be used to simulate buoyancy effects. A role could also be played by the limited response of the FFID instrumentation (response time $5 \mathrm{~ms}$ ). The dimensionless frequency response of the FFID is equivalent to $\mathrm{n} \sim 30-40$ (taking $\mathrm{f}=150$ $200 \mathrm{~Hz}$ ) and therefore might well be the main reason for the rapid roll-off in the spectra. Note also that the spectral peak lies between $n=0.1$ and 1.0 in the velocity spectra (Fig. 2c) but is much broader in the concentration spectra, being from $n=0.1$ to 10 . This difference is mirrored in the spectra presented in Fackrell and Robins [7].

The results shown in Fig. 6a for the single plume at $\mathrm{x} / \mathrm{H}=0.1$ and $\mathrm{y}=0$ indicate a significant difference in the shape of the PDF at the three locations, with larger $\chi$ being more frequent on the lower and stably stratified side of the plume. The power spectra in Fig. $6 \mathrm{~b}$ $(\mathrm{x} / \mathrm{H}=0.1, \mathrm{y}=0)$ clearly show that the high frequency contributions are reduced in the upper part of the plume relative to the centre and the lower part. However, the low frequency contribution is greater and this implies that the characteristic time scales of concentration 

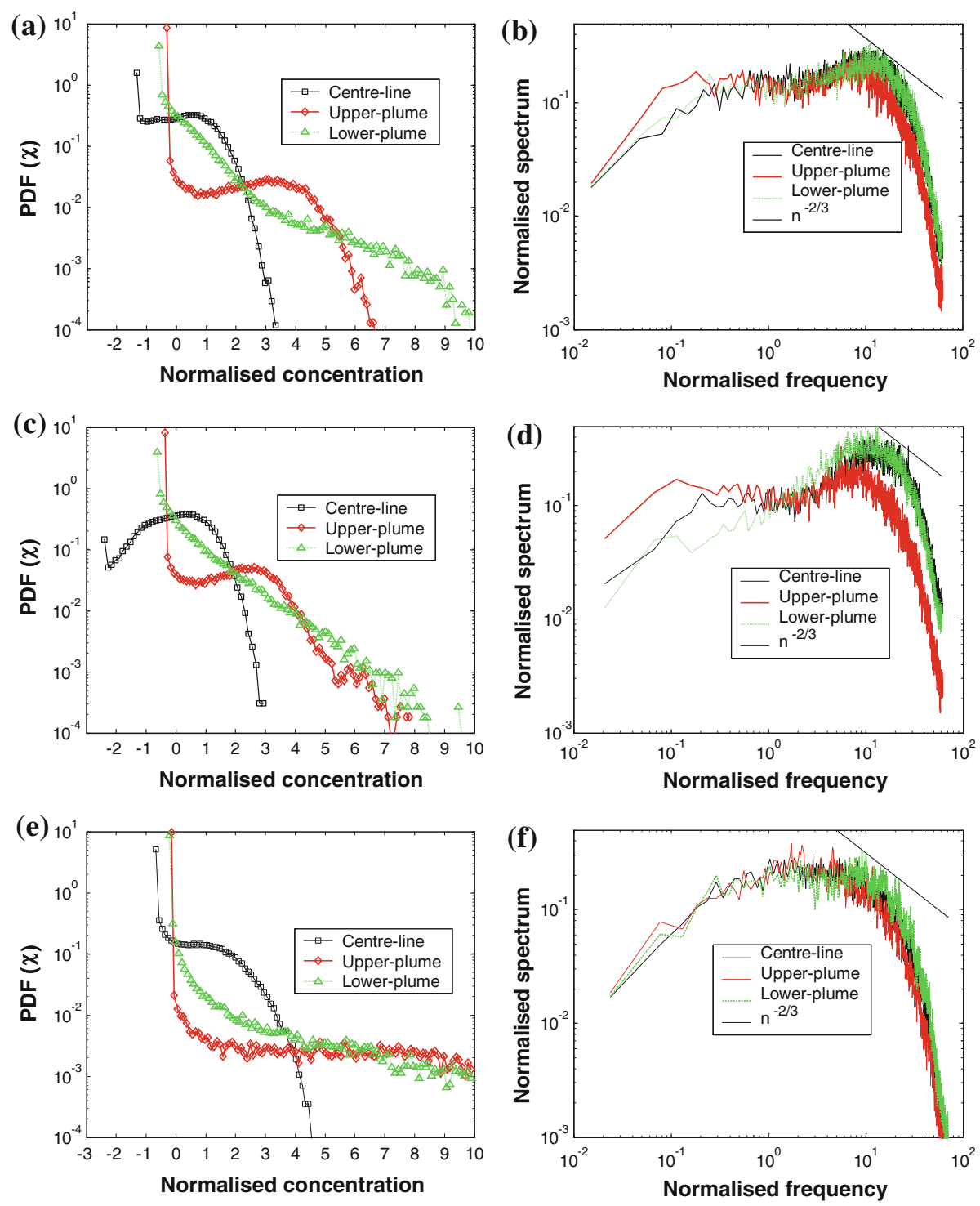

Fig. 6 PDFs and power spectra of concentration fluctuations on the plume centre-line and at the lower and upper edges of the plume for selected cases. a PDFs for a single plume at $\mathrm{x} / \mathrm{H}=0.1$. b Power spectra for a single plume at $\mathrm{x} / \mathrm{H}=0.1$. $\mathrm{c}$ PDFs for twin plumes and $\phi=0^{\circ}$ at $\mathrm{x} / \mathrm{H}=0.1$. d Power spectra for twin plumes and $\phi=0^{\circ}$ at $\mathrm{x} / \mathrm{H}=0.1$. e PDFs for twin plumes and $\phi=0^{\circ}$ at $\mathrm{x} / \mathrm{H}=0.6$. f Power spectra for twin plumes and $\phi=0^{\circ}$ at $\mathrm{x} / \mathrm{H}=0.6$

fluctuations are larger in the upper part of the plume than in the lower. In Fig. 6c, d the PDF and the power spectra are plotted at $\mathrm{x} / \mathrm{H}=0.1$ in the centre-plane of the combined plume $\left(\mathrm{y}=\mathrm{y}_{\text {mid }}\right)$ from two sources with $\phi=0^{\circ}$. Equivalent results at $\mathrm{x} / \mathrm{H}=0.6$ are shown in Fig. 6e, f. Vertical asymmetry is clearly also present in the two-source configuration and is actually enhanced by the mixing process at small values of $\mathrm{x} / \mathrm{H}$ (i.e. $\mathrm{x} / \mathrm{H}=0.1, \mathrm{x} / \mathrm{D}=20$ $\left.\phi=0^{\circ}\right)$. The asymmetry remains apparent at $\mathrm{x} / \mathrm{H}=0.6(\mathrm{x} / \mathrm{D}=120)$ but has almost disap- 
(a)
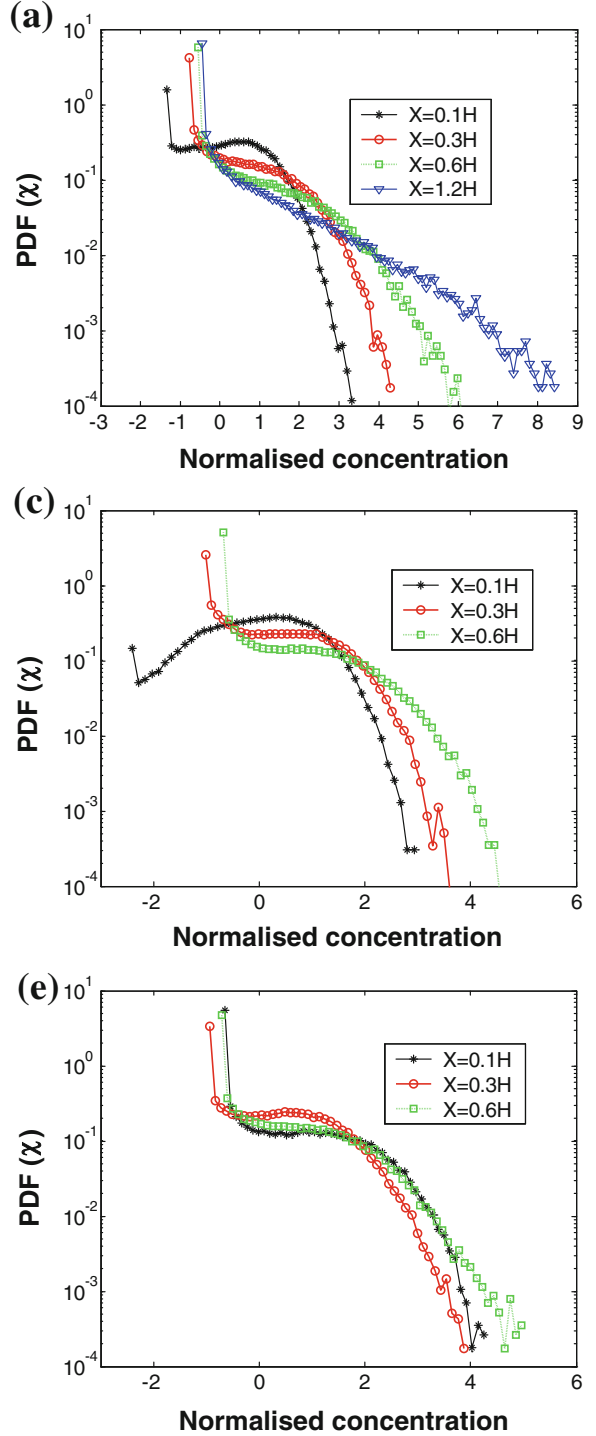
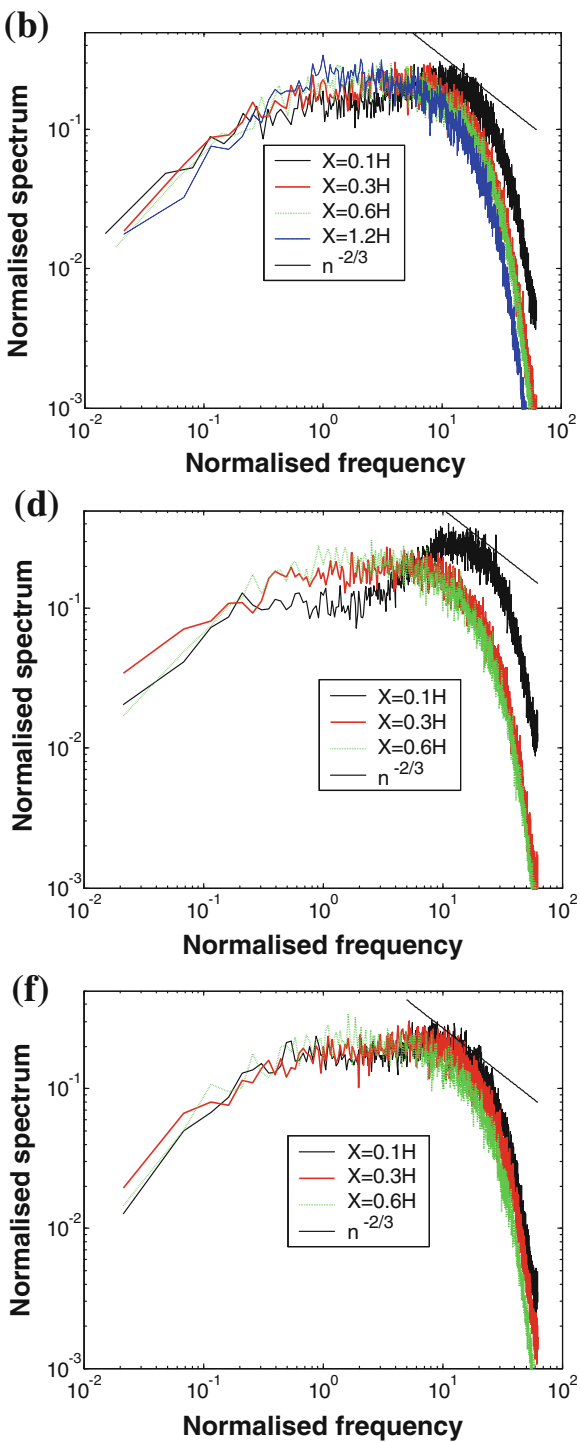

Fig. 7 PDFs and power spectra of concentration fluctuations on the plume centre-line for selected cases. a PDFs for a single plume. b Power spectra for a single plumes. $\mathbf{c}$ PDFs for twin plume and $\phi=0^{\circ}$. $\mathbf{d}$ Power spectra for twin plumes and $\phi=0^{\circ}$. e PDFs for twin plumes and $\phi=90^{\circ}$. f Power spectra for twin plumes and $\phi=90^{\circ}$

peared at $\mathrm{x} / \mathrm{H}=1.2(\mathrm{x} / \mathrm{D}=240)$ downwind (not shown). Taken together, the PDF results show more exponential-like distributions at the plume edge, particularly the lower edge, than in the plume centre. The intermittency, $\mathrm{I}$, is small at the plume centre at $\mathrm{x} / \mathrm{H}=0.1$, which favours clipped-Gaussian-like forms, but is much larger at the edges, favouring exponential forms $[6,7,40,41]$.

The development of the PDF and power spectrum with increasing downwind fetch along the plume centreline is demonstrated by the selection of results plotted in Fig. 7 for a single 
plume and for two-source configurations with $\phi=0^{\circ}$ and $90^{\circ}$. The results confirm the trend discussed above, in that the PDF evolves towards an exponential-like form (for non-zero concentrations) as x increases, as also argued by Csanady [3] and seen in the results reported, for example, in Yee et al. [4]. The development of the PDF is similar for the single and two-source plumes, though the approach to the exponential-like shape appears to be slower in the latter cases. As previously mentioned, this development is associated with an increase in I on the centre-line and, again, we emphasise that the results apply to a near-field region where the plume is becoming more intermittent as it is carried away from its source. Further downwind, especially in the upper and lower parts of the plume, near asymptotic behaviour is observed.

The associated power spectra, plotted as $\mathrm{fS}_{\mathrm{c}} / \sigma_{\mathrm{c}}^{2}$, show a clear peak (in an otherwise broad maximum) around a normalised frequency of $\mathrm{n}=1$, at small $\mathrm{x}$, that shifts towards lower frequencies as $\mathrm{x}$ increases and merges into the general spectrum form at the farthest downwind location $(\mathrm{x} / \mathrm{H}=0.6)$. This peak is not observed in the wind velocity power spectra, so it is reasonable to associate it with the turbulence internally generated within the plume. This is in agreement with the observations reported in Hanna [37], where two characteristic scales were assumed to be important in concentration fluctuations, and the integral time scale of concentration fluctuations within the plume was lower that that of the turbulent flow in which the plume was immersed. It should be noted that the general features of the PDFs and spectra in Figs. 6 and 7 were also observed in the non-buoyant emissions. Of course, these too were 'bent-over' plumes and much of the structure observed in the experiments derives from the interaction between the upwards emission, buoyant or not, and the cross-flow. However, buoyancy effects, when strong, may well modify or even determine the scale and intensity of the concentration fluctuations. The important structural difference, relative to passive dispersion, is the counter-rotating vortex pair that arises as a consequence of either upwards momentum or buoyancy in the emission. A truly passive release is generally produced in wind tunnel work by a horizontally directed released with an emission velocity equal to that of the local ambient flow and consequently generates a plume without an imbedded vortex pair.

For all the single plume cases, a fourth-order polynomial was fitted to the spectrum to obtain the frequency, $\mathrm{f}_{\max }$, of the spectral peak and a corresponding length scale, $\mathrm{L}$, evaluated as $\mathrm{L}=\mathrm{U}(\mathrm{z}) / \mathrm{f}_{\max }$. The scale $\mathrm{L}$ was then compared with the geometric radius $\mathrm{R}_{\mathrm{P}}$ of the plume, defined as $R_{P}=0.5 \sqrt{D_{Y} D_{Z}}$, where $D_{Y}$ and $D_{Z}$ represent, respectively, the horizontal and vertical diameters of the plume. These were empirically calculated from the measured concentration maps, as the vertical and horizontal extension of the contour at which the concentration was $10 \%$ of the maximum observed in a whole map. The relation between $\mathrm{L}$ and $R$ in a single plume is plotted in Fig. 8a, showing $L=R_{p}$ for $R_{p}$ less than about $100 \mathrm{~mm}$ (i.e. for relatively narrow plumes near the source, up to about $\mathrm{x} / \mathrm{H}=0.3$ ) indicating that the spectra peaks correspond to eddies or vortices with a size equal to about half of the plume diameter, which is precisely the length scale of the vortices in the counter-rotating vortex pair. At greater distances downwind, $\mathrm{x} / \mathrm{H} \geq 0.6$, the concentration fluctuations are influenced by the external turbulence and the power spectra (not shown) gradually becomes similar to that of vertical wind velocity with the maximum at frequencies below those associated with the size of the plume itself.

Figure $8 \mathrm{~b}$ shows equivalent results for two-source cases with $\phi$ between $0^{\circ}$ and $90^{\circ}$. There is now more scatter in the data, with results for large values of $\phi$ being farthest from the $1: 1$ correspondence. It is clear in these cases that the spectral peak initially correspond to scales that are smaller than the radius of the merging plume, most obviously for $\phi=90^{\circ}$. The likely interpretation is that the eddy or vortex scale is related to that of the individual plumes, even during the mixing phase in which the identity of the plumes becomes blurred. This would 

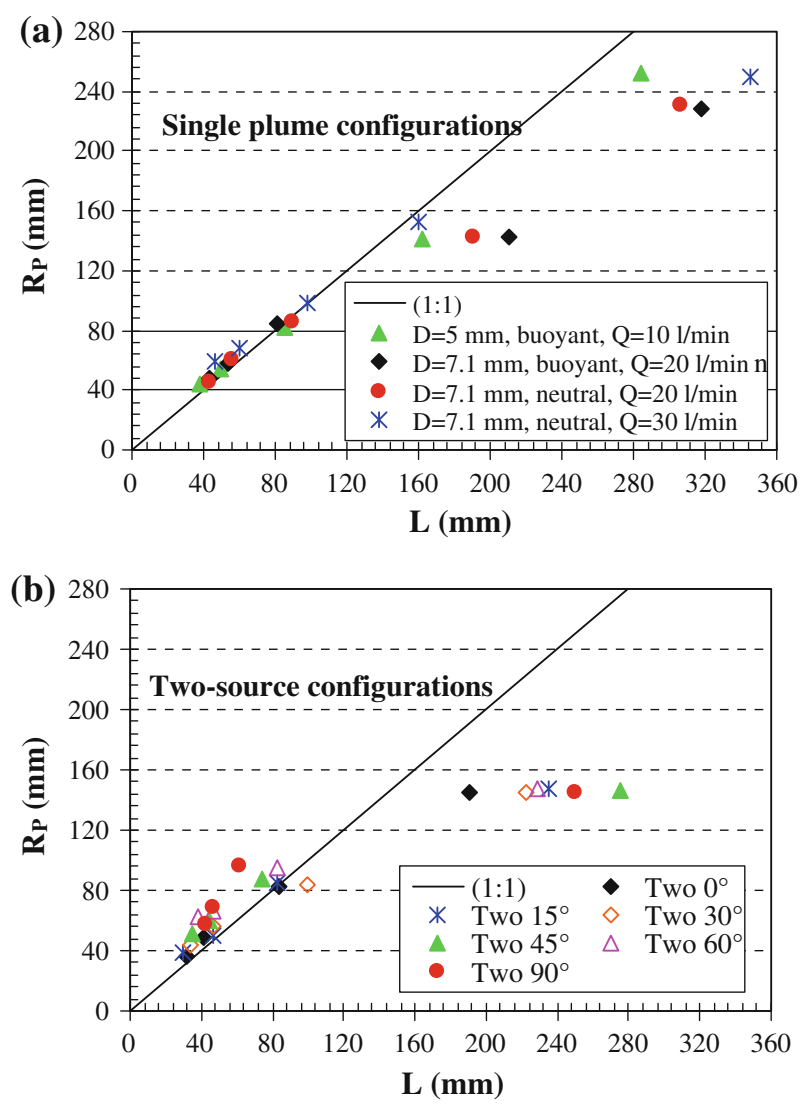

Fig. 8 Relationship between the plume radius and the length scale, L, associated with the peak in the power spectra. a Single plumes. b Twin plume cases. The figures include the 1:1 line

arise if the vortex system was the counter-rotating pairs associated with the individual plumes rather than a larger single vortex pair associated with the combined plumes. This conclusion follows the analysis reported in [23] in which it was shown how the presence of the vortex pair in each of the two merging plumes influences the shape of the combined plume and how this depends on the angle $\phi$. As already noted, at small values of $\phi$ the vortex pair guides the lower and more concentrated plume into the centre of the upper, less concentrated plume. For side-by-side plumes, at larger values of $\phi$, the interaction of two adjacent vortices (which have opposite signs of vorticity) distorts the plume, creating a "downwash" effect through a downward vertical velocity in the centre of the combining plume, relative to a centre of mass that is actually rising.

The preceding observations support some general comments on the phases of development in the plumes from both the single source and source pairs. Three phases of plume development emerge. The first occurs very close to the source (for $\mathrm{x} / \mathrm{H} \leq 0.3$ in the present cases) where the internally generated structure dominates the turbulence in the ambient flow. During this phase the concentration fluctuation power spectra have a broad peak at high frequency that appears to be associated with scales similar to the radius of the plume or, equivalently, to the size of the counter-rotating vortices. The peak frequency in the spec- 
tra does not increase significantly in the two-source configurations, even though the overall size of the combining plume increases, implying that during the first stage of the mixing the plumes maintain a degree of individual identity and the mixing takes place through the interaction of the vortex pairs that have developed within the individual plumes, thus generating fluctuations with characteristic scales significantly less than those of the combining plume (especially for large values of $\phi$ ). The second phase, at $\mathrm{x} / \mathrm{H} \geq 0.3$ is a transition phase in which there are significant contributions to the concentration fluctuations from both the turbulence internal to the plume and the external turbulence. The characteristics of the spectra and PDFs continue to evolve until the ambient turbulence becomes dominant. The third phase (for distances $\mathrm{x} / \mathrm{H}>0.6$ in the present case) is one in which the concentration fluctuations appear to be dominated by the external turbulence present in the ambient flow. In this phase, the power spectra tend to have a shape similar to that of the spectrum of the ambient vertical velocity fluctuations. Three key factors determine the extent of the three regions, the separation between the sources, the orientation of the sources to the oncoming wind and plume rise. The fetch to the region where the plumes begin to interact directly increases with the distance between the sources. This is also dependent on the orientation and is most pronounced for side-by-side sources, that is $\phi=90^{\circ}$. The final phase, dominated by ambient conditions, sets in once plume rise is essentially complete and, therefore, is established further downwind as emission momentum and buoyancy effects become stronger. It is important to note that in the two-source cases there were no appreciable changes in the spectral peaks of the concentration fluctuations even though the merging process slowed the final adjustment towards a form similar to the power spectrum of the vertical velocity fluctuations. This implies that the vortex pairs in each of the individual plumes continue as the dominant structures during the merging phase with the two plumes retaining a level of identity, rather than combining to create a coherent structure with a scale determined by the overall dimensions of the combined plumes.

\subsection{Concentration, variance, skewness and kurtosis}

Details of the development of the plume structure are more readily presented in terms of moments of the concentration signal rather than the PDF or power spectra themselves. Figure 9 shows the variation along the centre-line $\left(y=0, z=z_{p}\right.$ for the single source and $\mathrm{y}=\mathrm{y}_{\text {mid }}, \mathrm{z}=\mathrm{z}_{\mathrm{p}}$ for the source pair) of the dimensionless mean concentration, $\mathrm{C}^{*}$, the dimensionless variance, $\mathrm{V}$, skewness, $\mathrm{S}$, and kurtosis, $\mathrm{K}$. $\mathrm{C}^{*}$ is defined as:

$$
\mathrm{C}^{*}=\frac{\mathrm{CU}_{\mathrm{ref}} \mathrm{H}^{2}}{\mathrm{Q}}
$$

where $\mathrm{Q}$ is the combined emission rate. V, S and $\mathrm{K}$ are defined in Sect. 3.1. The dimensionless mean concentration, $\mathrm{C}^{*}$, plotted in Fig. 8 a for all buoyant plume cases, appears to collapse onto a single curve at distances of about $\mathrm{x} \geq 0.3 \mathrm{H}$. At smaller $\mathrm{x}$, concentrations associated with small values of $\phi\left(\phi \leq 15^{\circ}\right)$ are larger than those associated with $\phi \geq 30^{\circ}$, simply because in the latter case merging of the two plumes is slower because of their lateral separation and the concentration on the centre-line is therefore less. In interpreting the remaining results it should be remembered that they apply to the phase of development of a single plume in which it is becoming more intermittent as it moves away from its source, and therefore is in the phase where the dimensionless variance is increasing. This behaviour is compounded in the twin plumes by the interaction between the two, which depends on the orientation, $\phi$.

The variation of the normalised variance, $\mathrm{V}$, is shown in Fig. 9b, the skewness, $\mathrm{S}$, in Fig. 9c and the kurtosis, K, in Fig. 9d. These all show a similar behaviour to $\mathrm{C}^{*}$; for the twin 

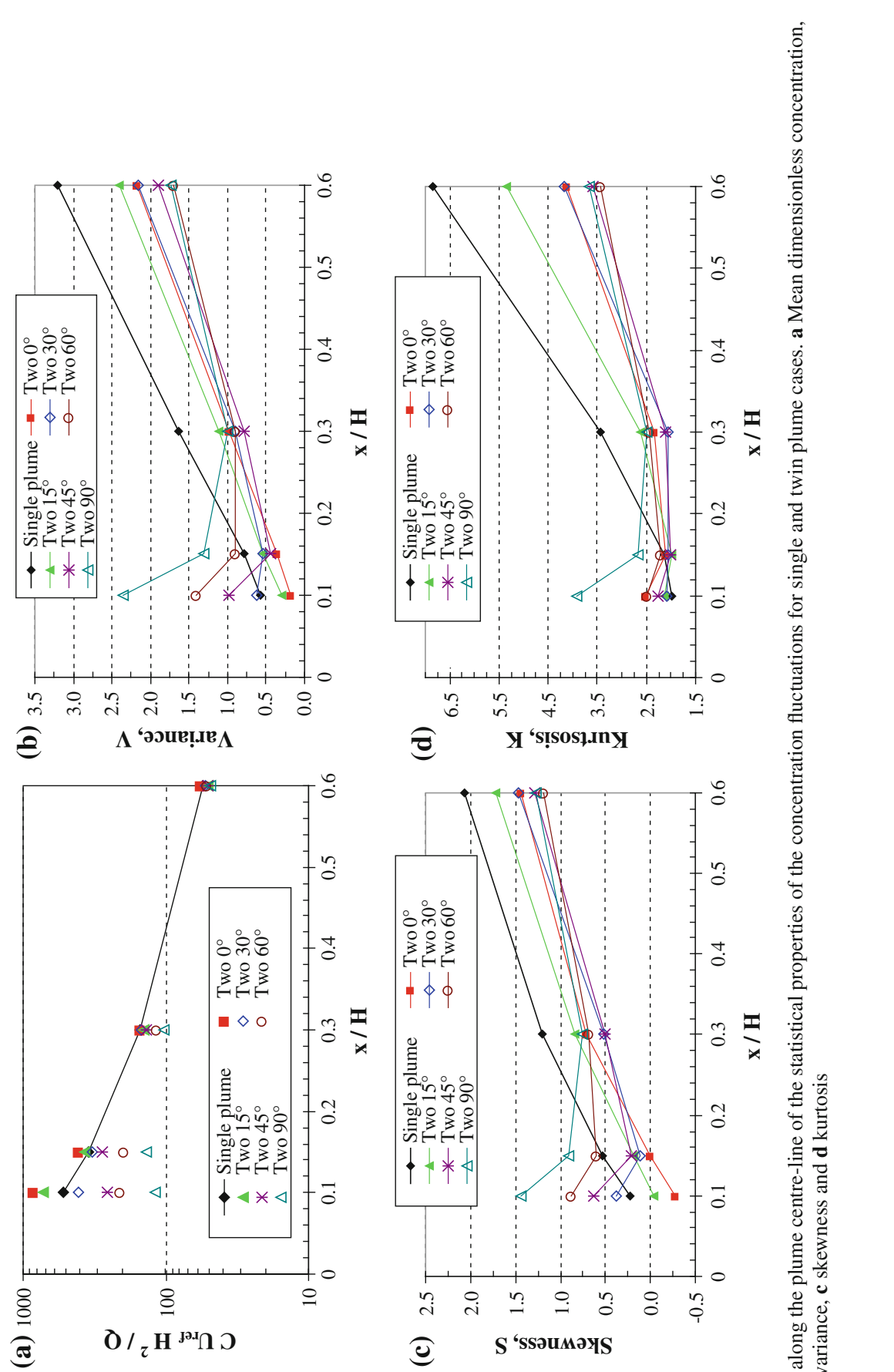
plumes reflecting the merging process for $\mathrm{x} / \mathrm{H}<0.3$ and then converging further downwind. However, there are distinct differences between the results for the single plume and the plume pairs. There is a clear dependence on the orientation, $\phi$, in the results for $\mathrm{V}, \mathrm{S}$, and $\mathrm{K}$. In plumes that are closely aligned with the approaching flow, $\phi \leq 15^{\circ}$, all three properties increase steadily with increasing fetch, $\mathrm{x}$, The same is seen in the single plume but in this case V, S, and $\mathrm{K}$ are all somewhat larger. This can be interpreted as reflecting the smaller size of the single plume which, in passive dispersion, would result in exactly this behaviour. For all other values of $\phi$ the merging process results in a decrease in V,S, and $\mathrm{K}$ to minima between $\mathrm{x} / \mathrm{H}=0.15$ and 0.3 , followed by steady growth, in line with the cases for $\phi \leq 15^{\circ}$. The most likely explanation is that for $\mathrm{x} / \mathrm{H}<0.3$ the results reflect conditions in the outer parts of the individual plumes, rather than the centre of a combined plume. The greater the lateral separation of the plumes, the greater the fetch before merging takes place, reflected in the minima of $\mathrm{V}, \mathrm{S}$ and $\mathrm{K}$ being furthest downstream, at $\mathrm{x} / \mathrm{H}=0.3$, for $\phi=90^{\circ}$.

Vertical profiles of $\mathrm{V}, \mathrm{S}$ and $\mathrm{K}$ at $\mathrm{y}=\mathrm{y}_{\text {mid }}$ for two-source cases and $\mathrm{y}=0$ for the single plume are shown in Fig. 10 at $\mathrm{x} / \mathrm{H}=0.1$ and 0.6 . Firstly, all three properties increase towards the plume edges, just as in passive dispersion, reflecting the increased intermittency there. Trends with orientation are most obvious in the variance, particularly at $\mathrm{x} / \mathrm{H}=0.1$. In the lower part of the plumes there is a clear increase in the variance with $\phi$ (note that the scale is logarithmic) and this reflects the varying degrees of merger in the plume pairs; similar, though lesser, trends are seen in the results for $\mathrm{S}$ and $\mathrm{K}$. Interpretation of the data further downwind, at $\mathrm{x} / \mathrm{H}=0.6$, is made more difficult by the different degrees of plume rise, as witnessed by the locations of the minima in, say, V (see also Fig. 3 in [30]). The plume pairs with $\phi=0$ and $15^{\circ}$ are the most affected. Plume rise is much less at $\mathrm{x} / \mathrm{H}=0.1$ and consequently less of an issue in this respect. Another feature of Fig. 10a is that the differences due to orientation of the plume pairs, which has a great impact on the variance on and below the centre-line, has relatively little effect in the upper part of the plumes, something that is reflected to a lesser degree in Fig. 10c, e. Mean concentration cross sections of merging plumes are shown in Fig. 5 in [30], for $\phi=15^{\circ}$ and Fig. 6, for $90^{\circ}$. In the former case, a near-classical vortex-pair structure appears to have been established at $\mathrm{x}=447 \mathrm{~mm}(\mathrm{x} / \mathrm{H}=0.45)$, but not in the latter case. Perhaps of more interest are the cross sections for $\phi=90^{\circ}$ at $\mathrm{x}=130$ and $305 \mathrm{~mm}$ $(\mathrm{x} / \mathrm{H}=0.13$ and 0.31$)$. The most obvious difference in the structure at these locations relative to a single or well merged plume is the 'bulge' in the central, lower region. This is where two vortices of opposite sign (one from each of the two adjacent vortex pairs) are interacting, which is likely to be a rather unstable process and the reason for the differences noted, for example, in the variance profiles in Figure 10a.

Equivalent profiles in the crosswind direction in single and merged plumes are plotted in Fig. 11. The measurements showed that concentration fluctuations were numerically at a maximum on the plume centre-line but as they did not decay with crosswind distance as rapidly as the mean concentration, the ratio $\sigma_{\mathrm{c}} /\langle\mathrm{c}\rangle$ increases towards the plume edges, a well established feature of plume structure (e.g. see [6,7,15]). As in Fig. 4e, the inefficient merging of plumes when $\phi \geq 45^{\circ}$ leads to a peak in the $\mathrm{V}, \mathrm{S}$ and $\mathrm{K}$ profiles on the centre-line $\left(\mathrm{y}=\mathrm{y}_{\text {mid }}\right)$. By way of contrast, there is very little difference between the profiles in a single plume and in a pair of plumes when $\phi=0^{\circ}$.

\section{Conclusions}

In this work some statistical properties of the concentrations fluctuations in two merging, buoyant plumes are discussed and analysed by comparing their behaviour with that observed 

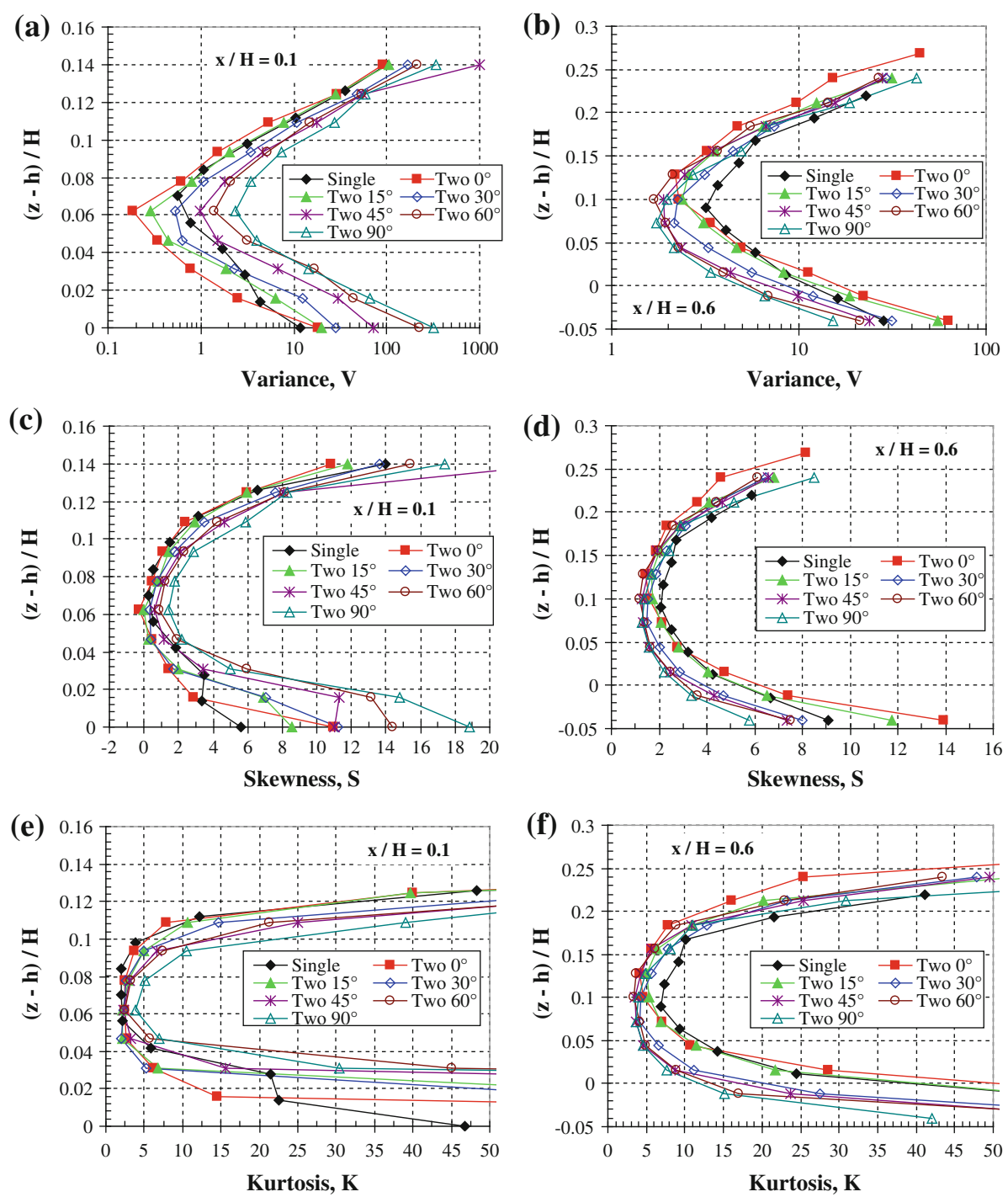

Fig. 10 Vertical profiles of statistical properties in the plume centre-plane $\left(y=y_{\text {mid }}\right)$ for single and twin plume cases. a Normalised variance, $V$, at $x / H=0.1$. b x/H=0.6. c Skewness, $S$, at $x / H=0.1$. d x/H=0.6. e Kurtosis, $\mathrm{K}$, at $\mathrm{x} / \mathrm{H}=0.1$ and $\mathbf{f} \mathrm{x} / \mathrm{H}=0.6$

in a single developing plume and also in neutrally buoyant dispersion. The analysis, based on wind tunnel concentration data, refers to the first four moments of the concentration field, and to the intermittency, the probability density function and the power spectrum. Results for a single plume showed asymmetry in vertical profiles of the four moments in both buoyant and neutral emissions due to the internal structure that develops as the emission is bent-over in the cross flow (creating a counter-rotating vortex pair). This was also the case for the plume pairs but made more complex by the dependence of the interaction on the alignment of the plumes with the approaching flow. This asymmetry was also observed in the intermittency as well 

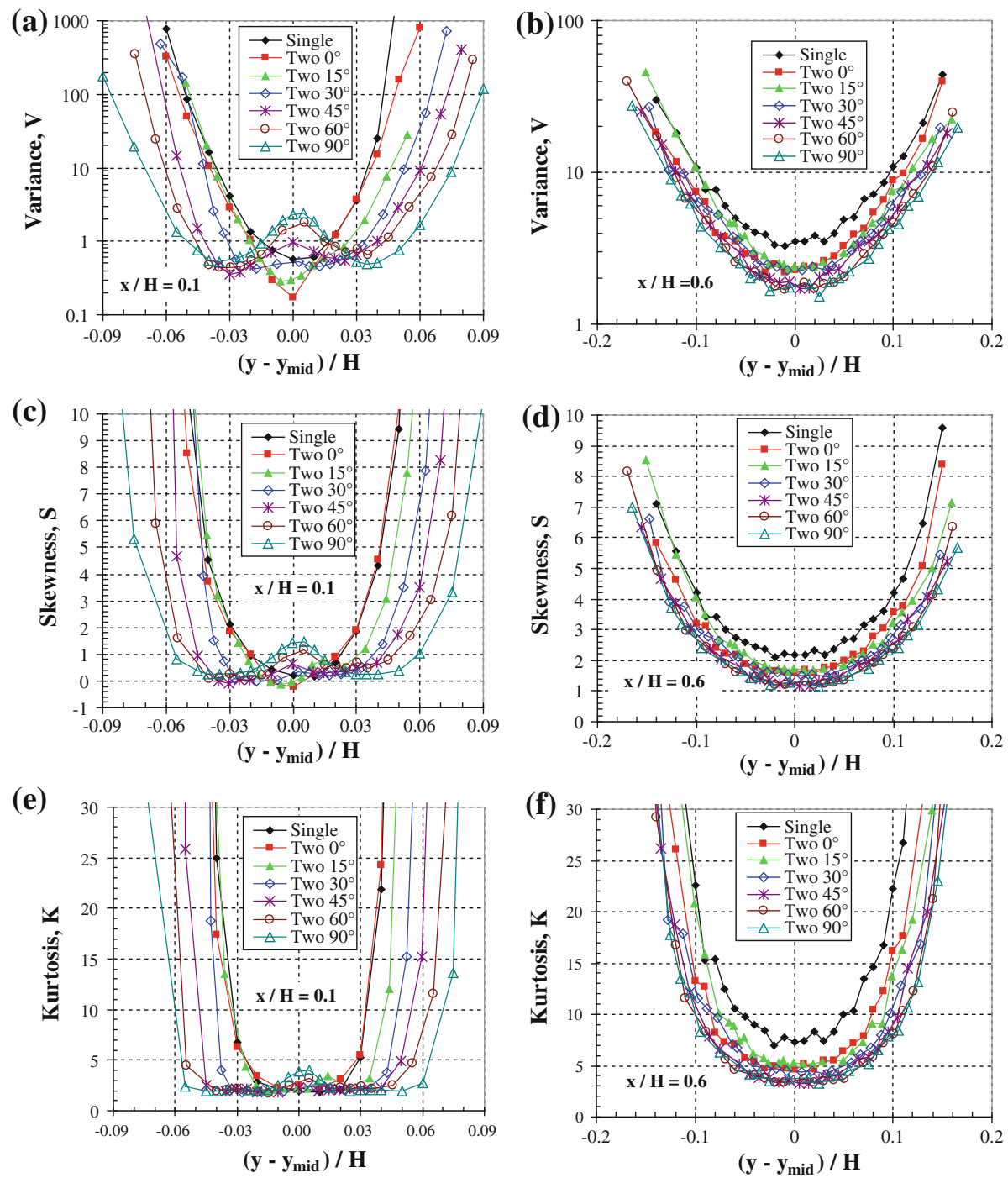

Fig. 11 Horizontal profiles of statistical properties at the plume heigth $\left(\mathrm{z}=\mathrm{z}_{\mathrm{p}}\right)$ for single and twin plume cases. a Normalised variance, $\mathrm{V}$, at $\mathrm{x} / \mathrm{H}=0.1$. b $\mathrm{x} / \mathrm{H}=0.6$. c Skewness, $\mathrm{S}$, at $\mathrm{x} / \mathrm{H}=0.1$. d x/H=0.6. e Kurtosis, $\mathrm{K}$, at $\mathrm{x} / \mathrm{H}=0.1$ and $\mathbf{f} \mathrm{x} / \mathrm{H}=0.6$

as in the PDFs and in the power spectra of the concentration fluctuations. Additionally and for geometrical reasons, the mixing process can induce horizontal asymmetry, not present in a single plume, depending on the alignment angle $\phi$.

The analysis identified three phases in the plume development in both neutral and buoyant plumes. The first, very near the stack, is dominated by turbulence generated within the plume, with concentration spectra showing distinct peaks that correspond to length scales that are comparable with the size of the vortex pairs in the individual plumes. A second phase follows at somewhat greater distances downwind where there are significant contributions to the concentration fluctuations from both the turbulence internal to the plume and the external 
turbulence. This is a transition phase in which the shapes of the spectra and the PDFs are evolving. A third phase (for distances $\mathrm{x} / \mathrm{H}>1$ in the cases studied here) then follows in which the concentration fluctuations appear to be governed by the external turbulence present in the ambient flow. In this phase, the power spectra tend to have a shape similar to that of the ambient vertical velocity fluctuations. The merging of the two plumes mainly affects the first phase by controlling its extent in time and space through the interaction of the counterrotating vortex systems of the two individual plumes. Results show that the vortex pairs of the individual plumes continue to be the dominant structure in the mixing phase where the two plumes retain a degree of individual identity and the scales remain those of the vortex pairs rather than the overall dimensions of the combined concentration field. In general, the extent of the three phases is determined by the distance between the sources, their orientation to the approaching flow and plume rise, the latter dependent on both emission properties and the cross flow conditions.

The intermittency, variance, skewness and kurtosis along the centre-lines of the plume pairs were consistently less than found in a single plume for $\mathrm{x} / \mathrm{H}>0.3$ (in the cases studied) but the variation with $\mathrm{x}$ was similar. However, for $\mathrm{x} / \mathrm{H}<0.3$ there was a clear variation in these parameters with orientation, $\phi$, that is related to the merging process between the vortex systems in the plume pairs and leads to a local minimum in the observed properties when $\phi \geq 30^{\circ}$. In these cases, the property differences are mainly found in the centre and lower sections of combined plumes with only minimal differences in the upper parts. These features remained at the farthest downwind location studied, $\mathrm{x} / \mathrm{H}=0.6$, and, though diminished, they represent a structural modification of the concentration field in the combined plume, again associated with the role of the vortex pairs in the mixing process. It is the presence of the vortex pair in both the buoyant and neutral plumes that is mainly responsible for the differences between these classes of plume and that from a truly passive emission. Although the internal structure of plumes with significant buoyancy and upwards momentum is dominated by the counter-rotating vortex pair, the form of their development downstream from their source is not that dissimilar to that found in passive dispersion. The work described here treated the phase in which plume rise controlled the plume behaviour. This was also the region of development in which intermittency increased and the intensity of concentration fluctuations did likewise. Whether the plume rise, intermittency and fluctuation always develop on a common scale is something that needs to be investigated as in passive dispersion the only source property of relevance is the source size. There is also the question of behaviour once plume rise is essentially complete and how this matches with passive dispersion. Further experiments and investigations are needed to address these questions.

Acknowledgments This research was carried out with partial financial support from the mobility funds of the CNR-NATO "Senior Fellowship" program. The authors wish to thank Mr. Allan Wells, of the University of Surrey, for his help in setting up the experiments.

\section{References}

1. Carpentieri M, Kumar P (2011) Ground-fixed and on-board measurements of nanoparticles in the wake of a moving vehicle. Atmos Environ 45:5837-5852

2. Hinds WC (1999) Aerosol technology. Properties, behaviour, and measurement of airborne particles, 2nd edn. Wiley, New York

3. Csanady GT (1967) Concentration fluctuations in turbulent diffusion. J Atmos Sci 11:21-28

4. Yee E, Chan R, Kosteniuk PR, Chandler GM, Biltoft CA, Bowers JF (1994) Experimental measurements of concentration fluctuations and scales in a dispersing plume in the atmospheric surface layer obtained using a very fast response concentration detector. J Appl Meteorol 33:996-1016 
5. Klein PM, Young DT (2011) Concentration fluctuations in a downtown urban area. Part I: analysis of Joint Urban 2003 full-scale fast-response measurements. Environ Fluid Mech 11:23-42

6. Fackrell JE, Robins AG (1982a) The effects of source size on concentration fluctuations in plumes. Boundary Layer Meteorol 22:335-350

7. Fackrell JE, Robins AG (1982b) Concentration fluctuations and fluxes in plumes from point sources in a turbulent boundary layer. J Fluid Mech 117:1-26

8. Wilson DJ, Fackerell JE, Robins AG (1982a) Concentration fluctuations in an elevated plume: a diffusiondissipation approximation. Atmos Environ 16:2581-2589

9. Deardoff JW, Willis GE (1988) Concentration fluctuations within a laboratory convectively mixed layer. In: Venkatram A, Wyngaard JC (eds) Lecturers on air pollution modeling. American Meteorological Society, pp 357-384

10. Klein P, Leitl B, Schatzmann M (2011) Concentration fluctuations in a downtown urban area. Part II: analysis of Joint Urban 2003 wind-tunnel measurements. Environ Fluid Mech 11:43-60

11. Bara BM, Wilson DJ, Zelt BW (1992) Concentration fluctuation profiles from a water channel simulation of a ground level release. Atmos Environ 26A:1053-1062

12. Huq P, Stewart EJ (1997) Measurements of density fluctuations in steady, buoyant plumes in crossflow. Atmos Environ 31:1677-1688

13. Wilson DJ, Fackerell JE, Robins AG (1982b) Predicting the spatial distribution of concentration fluctuations from a ground level source. Atmos Environ 16:497-504

14. Sawford BL (1983) The effect of Gaussian particle-pair distribution functions in the statistical theory of concentration fluctuations in homogeneous turbulence. Q J R Meteorol Soc 190:339-354

15. Hanna SR (1984) Concentration fluctuations in a smoke plume. Atmos Environ 18:1091-1106

16. Yee E, Chan R (1997) A simple model for the probability density function of concentration fluctuations in atmospheric plumes. Atmos Environ 31:991-1002

17. Mortarini L, Franzese P, Ferrero E (2009) A fluctuating plume model for concentration fluctuations in a plant canopy. Atmos Environ 43:921-927

18. Mavroidis I, Andronopoulos S, Bartzis JG, Griffiths RH (2007) Atmospheric dispersion in the presence of a three-dimensional cubical obstacle: modelling of mean concentration and concentration fluctuations. Atmos Environ 41:2740-2756

19. Levellen WS, Sykes RI (1986) Analysis of concentration fluctuations from lidar observations of atmospheric plumes. J Clim Appl Meteorol 25:1145-1154

20. Jorgensen HE, Mikkelsen T, Pecseli HL (2010) Concentration fluctuations in smoke plumes released near the ground. Boundary Layer meteorol 137:345-372

21. Finn D, Clawson KL, Carter RG (2010) Analysis of urban atmosphere plume concentration fluctuations. Boundary Layer Meteorol 136:431-456

22. Pavageau M, Schatzmann M (1999) Wind tunnel measurements of concentration fluctuations in an urban street canyon. Atmos Environ 33:3961-3971

23. Contini D, Hayden P, Robins AG (2006) Concentration field and turbulent fluxes during the mixing of two buoyant plumes. Atmos Environ 40:7842-7857

24. Briggs GA (1974) Plume rise from multiple sources. In: Proceedings of the Cooling towers environment, Maryland, pp 161-179

25. Briggs GA (1984) Plume rise and buoyancy effects. In: Randerson D (ed) Atmospheric science and power production. US Department of Energy, DOE/TIC-27601, pp 327-366

26. Anfossi D, Bonino G, Bossa F, Richiardone R (1978) Plume rise from multiple sources: a new model. Atmos Environ 12:1821-1826

27. Bornoff RB, Mokhtarzadeh-Dehghan MR (2001) Numerical study of interacting buoyant cooling-tower plumes. Atmos Environ 35(3):589-598

28. Contini D, Robins A (2001) Water tank measurements of buoyant plume rise and structure in cross flow. Atmos Environ 35:6105-6115

29. MacDonald RW, Strom RK, Slawson PR (2002) Water flume study of the enhancement of buoyant rise in pairs of merging plumes. Atmos Environ 36:4603-4615

30. Contini D, Robins A (2004) Experiments on the rise and mixing in neutral crossflow of plumes from two identical sources for different wind directions. Atmos Environ 38:3573-3583

31. Kolar V, Savory E (2007) Dominant flow features of twin jets and plumes in crossflow. J Wind Eng Ind Aerodyn 95:1199-1215

32. Irwin HPAH (1981) The design of spires for wind simulation. J Wind Eng Ind Aerodyn 7:361-366

33. Foken T, Wichura B (1996) Tools for quality assessment of surface-based flux measurements. Agric For Meteorol 78:83-105 
34. Foken T, Gockede M, Mauder M, Mahrt L, Amiro B, Munger W (2004) Post-field data quality control. In: Lee X, Massman W, Law B (eds) Handbook of Micrometeorology_a guide for surface flux measurement and analysis. Kluwer, Netherlands, pp 181-208

35. Csanady GT (1973) Turbulent diffusion in the environment. Reidel, Boston, p 248

36. Sykes RI, Lewellen WS, Parker SF (1984) A turbulent-transport model for concentration fluctuations and fluxes. J Fluid Mech 139:193-218

37. Hanna SR (1986) Spectra of concentration fluctuations: the two time scales of a meandering plume. Atmos Environ 20:1131-1137

38. Mylne KR, Mason PJ (1991) Concentration fluctuation measurements in a dispersing plume at a range of up to $1000 \mathrm{~m}$. Q J R Meteorol Soc 117:177-206

39. Contini D, Cesari D, Donateo A, Robins AG (2009) Effects of Reynolds number on stack plume trajectories simulated with small scale models in a wind tunnel. J Wind Eng Ind Aerodyn 97:468-474

40. Yee E, Chan R (1997) A simple model for the probability density function of concentration fluctuations in atmospheric plumes. Atmos Environ 31:991-1002

41. Weil JC, Snyder WH, Lawson RE, Shipman MS (2002) Experiments on buoyant plume dispersion in a laboratory convection tank. Bound Layer Meteorol 102:367-414 